\title{
Ein haplo-diploides Zwillingspaar bei Triticum vulgare VILL.*
}

Von

\section{Yukio Yamamoto.}

\author{
Mit 26 Text-Figuren.
}

Eingegangen am \$3. Juli, 1936.

\section{Einleitung.}

Über haploide Pflanzen bei Triticum wurde bereits von Gaines und Aase (1926), Kinara und Katayama $(1932,1933)$, Katayama (1934a, b), Cinzaki (1934), Yamasaki (1934, 1935), Namikawa und Kawakami(1934), Naka.Jima (1935) und Kinara (1935) bereichtet. Im folgenden wird über eine haploide Pflanze bei T. vulgare mitgeteilt, die bei einem Zwillingspaar gefunden wurde; das andere Individuum des Paares war diploid. Die Erscheinung von haplo-diploiden Zwillingen wurde zuerst von KAPPENT (1933) bei Linum und, im gleichen Jahre, von Ramiah (et alii 1933) bei Reis beobachtet und näher beschrieben. Bei Triticum vulgare wurde sie von Namikawa und Kawakami (1934) festgestellt und bei T. durum von ḰIIIARa (1936).

\section{Material und Methoden.}

Das betreffende Zwillingspaar wurde bei einem in Kanto (Japan) verbreitetem Weizen (Saitama, Nr. 27) entdeckt.

Die Wurzelspitzen wurden mit LA-Coun's Flüssigkeit fixiert. Die in Dauerpräparaten untersuchten Blütenknospen wurden mit CARnoy-FlemMING nach der Vorschrift von TAHARA (1921) fixiert; für Schmierpräparate bediente ich mich der Eisenessigkarmin-Methode von Beluing. Die Dauerpräparate wurden mit Gentianaviolett nach NEwTon gefärbt. Die Dicke der Paraffinschnitte betrug bei den Blütenknospen $16 \mu$ und bei den Wurzeln $14 u$.

Die Beschaffenheit der Epidermis wurde nach "Suzuki's Universal Mikro-Printing Method,, (S.U.M.P.) untersucht.

* Contributions from the Laboratory of Gencties, Biological Institute, Kyoto Imperial University, No. 74. 


\section{Auftreten des haplo-diploiden Zwillingspaares.}

Aus ca. 3600 Körnern der genannten Weizensorte wurden 7 Zwillingspaare und 1 Drilling ${ }^{1)}$ gewonnen. Nur ein eiziges Paar war haplo-diploid.

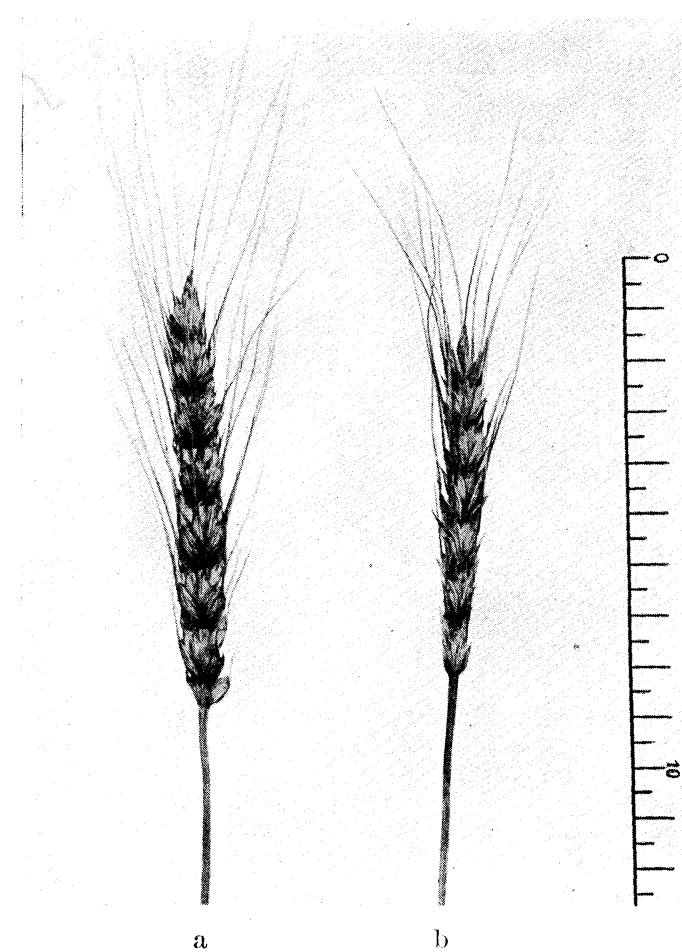

Abb. 1. Ähren von Haplo- und Diplovugare. a. Diplovulgare. b. Haplovulgare.

Der Grössenunterschied zwischen den beiden Individuen trat am deutlichsten nach Ausbildung der Ähren zutage. Die haploide Pflanze war bedeutend niedriger und wies kürzere und schmalere Ähren auf; auch ihre Blätter waren merklich schmaler als die der diploiden Pflanze (Abb. 1).

\section{Karyologische Befunde.}

In den Wurzelspitzen der haploiden Pflanze wurden 21 Chromosomen gezählt (Abb. 2). Während die somatische Chromosomengarnitur einer normalen diploiden Pflanze 4 zweifach eingeschnürte Chromosomen aufweist (Abb. 3), konnten bei der haploiden nur 2 solche Chromosomen gefunden werden.

In der I. Metaphase findet man meist 21 Univalente (Abb. 4); manchmal sieht man Chromosomenpaare, und zwar bis zu 3 (Abb. 5-7
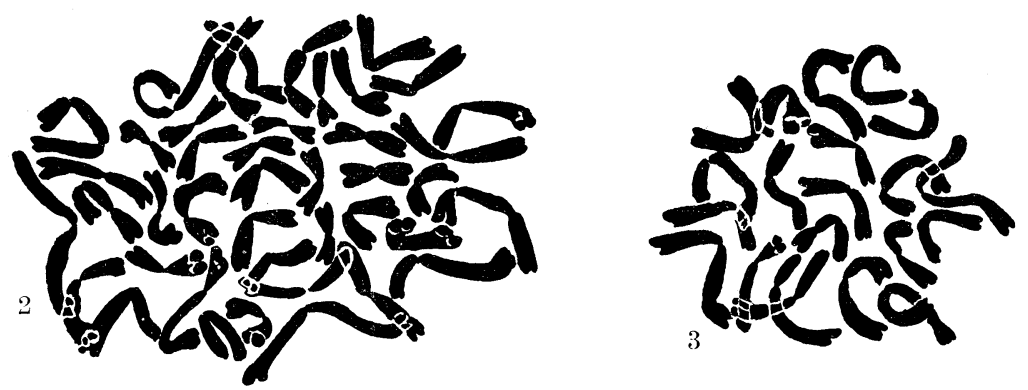

Abb. 2 und 3. Somatische Chromosomen aus Wurzelspitzen bei Haplo- und Diplovulgare. Vergr. ca. 2500-fache. Abb. 2. Diplovulgare. Abb. 3. Haplovulgare.

1) Triplo-triplo-diplodrilling (Näheres soll a. a. O. mitgeteilt werden). 

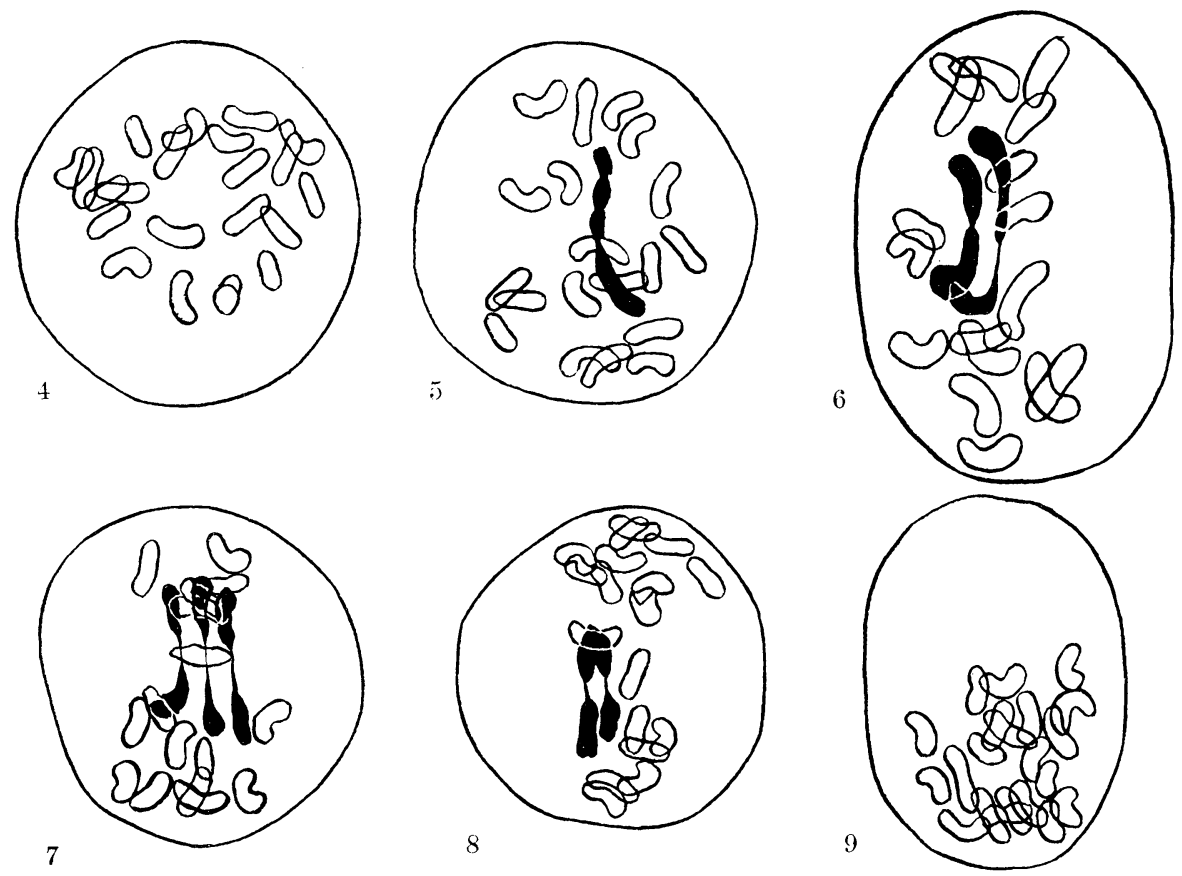

Abb. 4-19. Reifungsteilungen bei Haplovulgare. Vergr. ca. 2000-fach. Abb. 4-12. I. Metaphase. Verschiedene Konfigurationen.

Abb. 4. 21 . Abb. 5. $1_{\mathrm{II}}+19_{\mathrm{I}}$. Abb. 6. $2_{\mathrm{II}}+17_{\mathrm{I}} \cdot$ Abb. $7.3_{\mathrm{II}}+15_{\mathrm{I}}$.

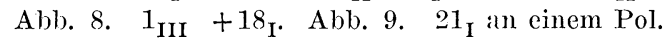

u. 21). Yamasaki konnte bis zu 4 Bipartiten in einem ähnlichen Falle beobachten. Vereinzelt kommen auch Tripartite vor ( $\Lambda$ bb. 8 u. 22). Über die Häufigkeit der Bindungen orientiert Tabelle 1. Die Bipartiten sind meistens locker gebunden. Abb. 10 bringt $6 \pm$ deutlich heteromorphe Bipartite von verschiedener Gestalt; mit Ausnahme von 10-f sieht man überall ein terminalisiertes Chiasma.

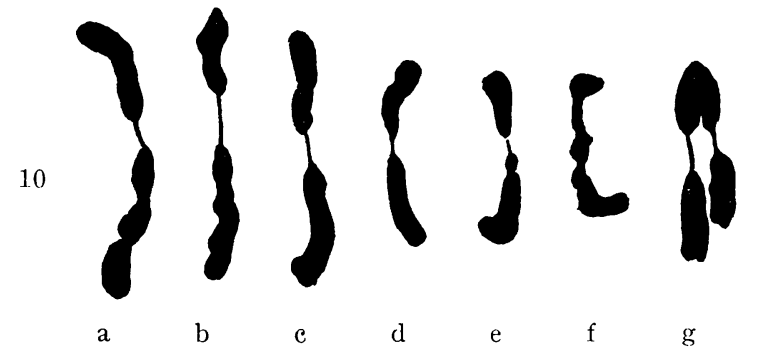

Abb. 10. 7 Bipartite von verschiedener Gestalt und 1 Tripartites aus verschiedenen Kernplatten in I. Metaphase.

Die Verteilung der Univalenten auf die Pole wurde an 300 P.M.Z. 
TABeLLe 1. Häufigkeit der I.M.Z. mit $0-3_{\text {II }}$ und $0-1_{I I I}$.

\begin{tabular}{|c|ccccc|c|}
\hline Bindungen. & 0 & $1_{\mathrm{I}}$ & $2_{\mathrm{II}}$ & $3_{\mathrm{II}}$ & $1_{\mathrm{III}}$ & Summe. \\
\hline Häufigkeit & 861. & 105 & 18 & 1 & 1 & 1000 \\
\hline$\%$ & 86.1 & 10.5 & 1.8 & 0.1 & 0.1 & 100 \\
\hline
\end{tabular}

TABELLE 2. Verteilung der Univalenten auf die Pole.

\begin{tabular}{|c|cccccccccccc|c|}
\hline Verteilung. & $0+21$ & $1+20$ & $2+19$ & $3+18$ & $4+17$ & $5+16$ & $6+15$ & $7+14$ & $8+13$ & $9+12$ & $10+11$ & Summe. \\
\hline beobachtet & 2 & 3 & 4 & 6 & 8 & 10 & 14 & 31 & 48 & 79 & 95 & 300 \\
\hline$\%$ & 0.7 & 1.0 & 1.3 & 2.0 & 2.7 & 3.3 & 4.7 & 10.3 & 16.0 & 26.3 & 31.7 & 100 \\
\hline theoretisch & 0.0001 & 0.00 & 0.02 & 0.1 & 0.6 & 1.9 & 5.2 & 11.1 & 19.4 & 28.0 & 33.6 & 100 \\
\hline
\end{tabular}

untersucht. Das Ergebnis ist aus Tabelle 2 erzusehen. Aus dem Vergleich mit den theoretischen Zahlen geht hervor, dass ihre Verteilung sich im grossen und ganzen zufallsmässig gestaltet. Im einzehnen sieht man aber Abweichungen. Die wichtigste besteht darin, dass die Grenzfälle $0+21$ und $1+20$ bedautend öfter vorkommen als erwartet. Die gleiche Erscheinung hat Kinara (1936) bei seinem Haplodurum festgestellt. Sie hängt höchst wahrscheinlich mit einem haufenweisen Transport der Univalenten zusammen, deren Ursache prophasische Endenverklebungen sein dürften. Manchmal wurde die Abwanderung eines Bipartiten nach einem Pol beobachtet (Abb. 11) (vgl. KIIIARa 1936). Hier und da begegnet man in früherer Metaphase drei poligen Figuren (Abb. 12). Die zum Äquator kommenden Univalenten teilen sich in der I. Netaphase längs, ähnlich wie bei Triticum-Bastarden, und die Spalthälften weichen nach den entgegengesetzten Polen auseinander. Regressionserscheinungen sind aber nicht selten, sowohl im ersten wie im zweiten Teilungsschriftt (Abb. 15). Auf dem Tetradenstadium findet man meist normal aussehende Pollentetraden (Abb. 18). Daneben begegnet man aber auch Pollendyaden, die durch Regression

Abl. 11. Frühere 1. Metaphase, $8+13$; 2 punktierte Chromosomen zeigen Endenverklebung.

Abb. 12. Dreipolige Metaphase.

Abb. 13. I. Anaphase; 3 Univalente ini Äquator.

Abb. 14. I. Telophase; 3 verzögerte Univalente längsgeteilt.

Abb. 15. Bildung eines Restitutionskerns in I. Anaphase.

Abb. 16. Interkinese; 3 Kernen in einer Tochterzelle und 1. Kern in der anderen.

Abb. 17. II. Anaphase mit 5 bzw. 3 verzögerten Monaden.

Abb. 18. Eine Pollentetrade.

Abb. 19. Eine Pollendyade.

Abl). 20. Pollenkörner von Haplovulgare. a. Äusserlich normales Korn. b. geschrumpfte. inhaltsarme Pollenkörner; auch das kleinste Pollenkorn hat eine Keimpore. Vergr. ca. 730 -fach. 

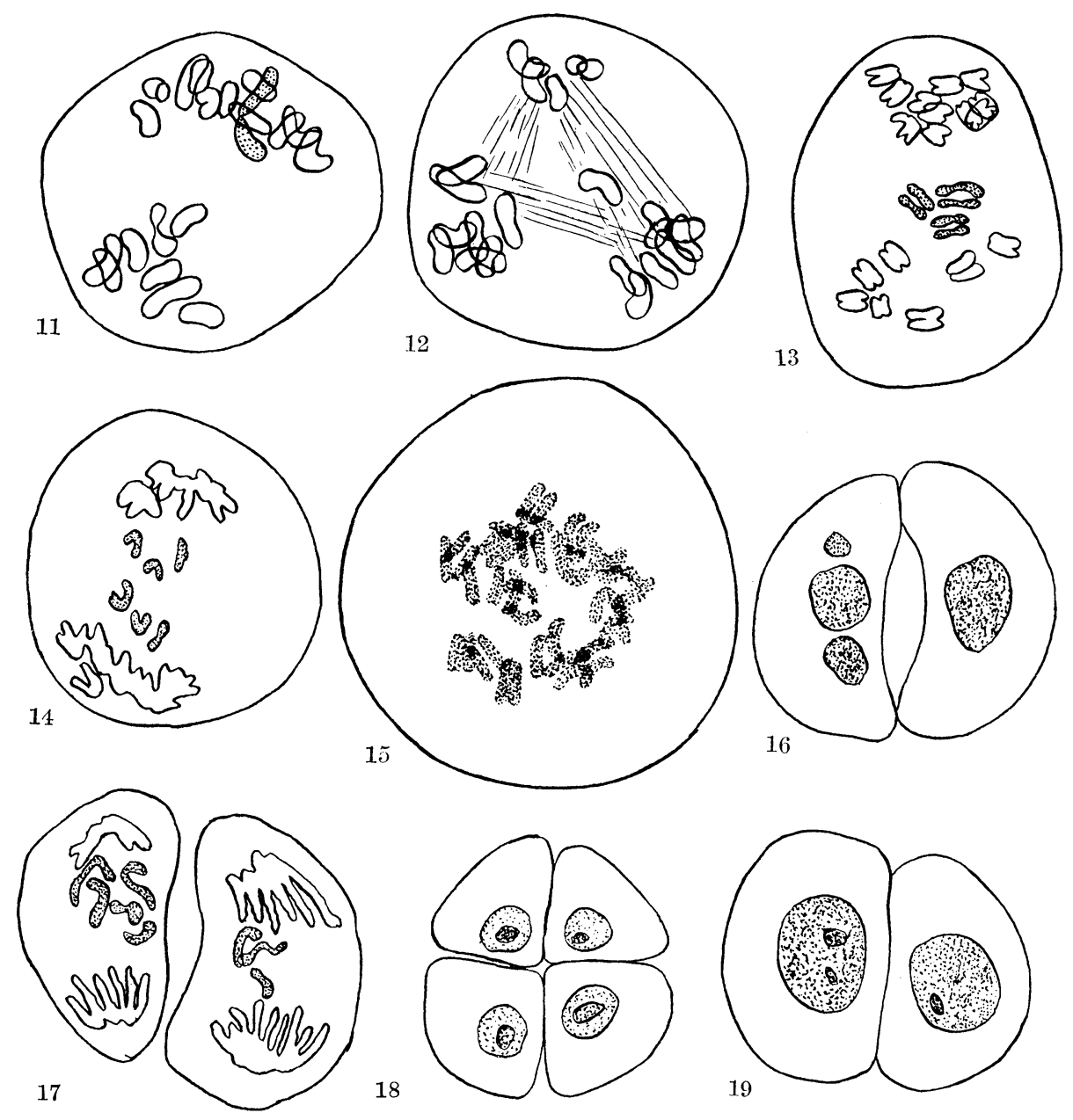

18
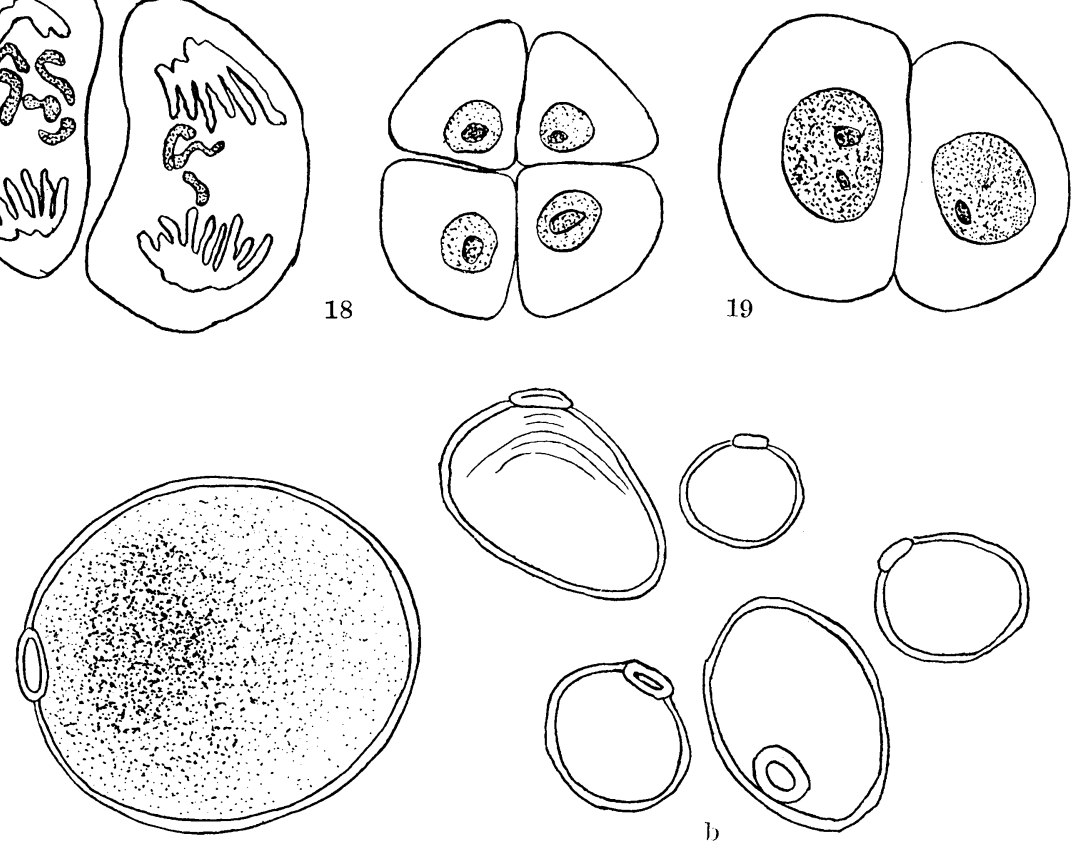


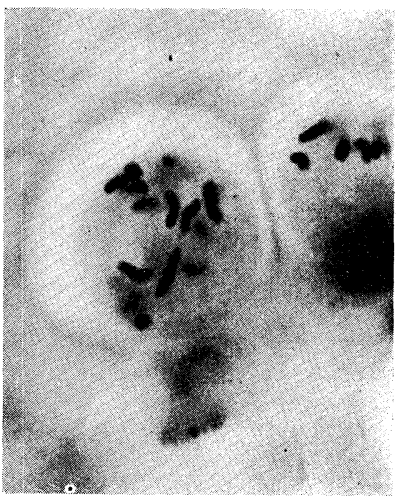

21

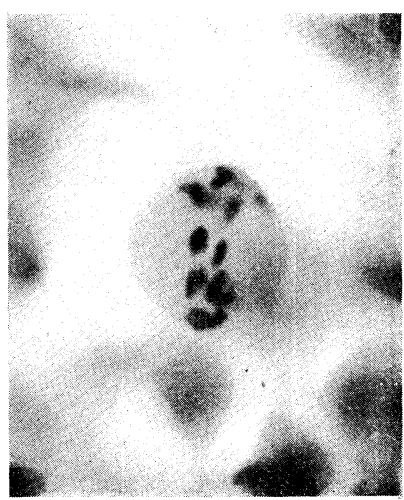

22

Abb. 21-22. Mikrophotographien von P. M. Z. ron Haplovulgare in I. Metaphase. Vergr. ca. 630-fach. Abb. 21. $1_{\mathrm{II}}+19_{\mathrm{I}}$. Abb. 22. $1_{\mathrm{III}}+18_{\mathrm{I}}$.

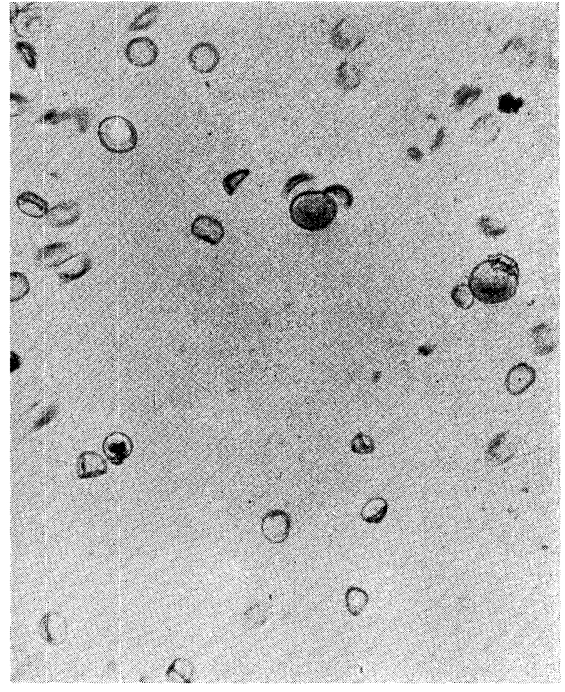

23

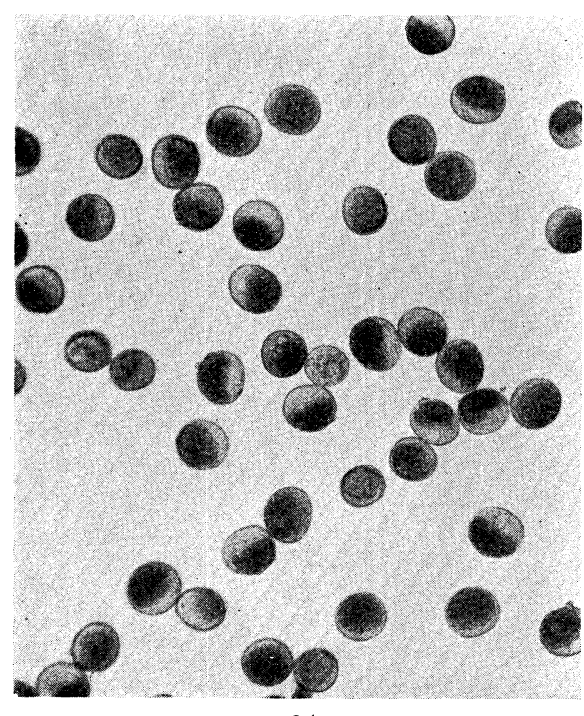

24

Abb. 23. Mikrophotographie. Pollenkörner ron Haplovulgare. Vergr. ca. 90-fach.

Abb. 24. Mikrophotographie. Pollenkörner ron Diplovulgare, Vergr. ca.90-fach.

entstanden sind, sowie Triaden, Pentaden und Hexaden (Abb. 19). In späteren Stadien verlieren die Pollenkörner die regelmässige Gestalt und erscheinen meist leer. Nur vereinzelt sieht man äusserlich taugliche Pollenkörner, die durch Regression entstanden sein dürften (Abb. 20a u. 23). Auf 1000 Pollenkörner konnten nur 7 tauglich aussehende gefunden werden (Tab. 3). Sämtliche Pollenkörner weisen eine Keimpore auf (Abb. 20-b).

\section{Epidermisbeschaffenheit.}

Wie Abb. 25 und 26 zeigen, sind die Zellelemente der Epidermis bei 
Haplovulgare merklich kleiner als bei Diplovulgare.

TABelle 3. Häufigkeit der äusserlich normalen und inhaltsaimen Pollenkörner.

\begin{tabular}{|c|c|c|c|}
\hline Pollenkategorien. & äusserlich norm. & inhaltsarm. & Summe. \\
\hline beobachtet & 7 & 993 & 1000 \\
\hline$\%$ & 0.7 & 99.3 & 100 \\
\hline
\end{tabular}

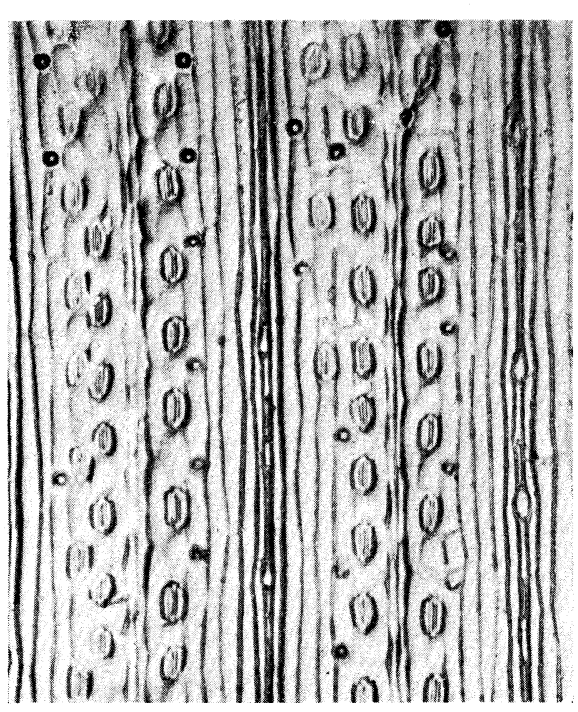

25

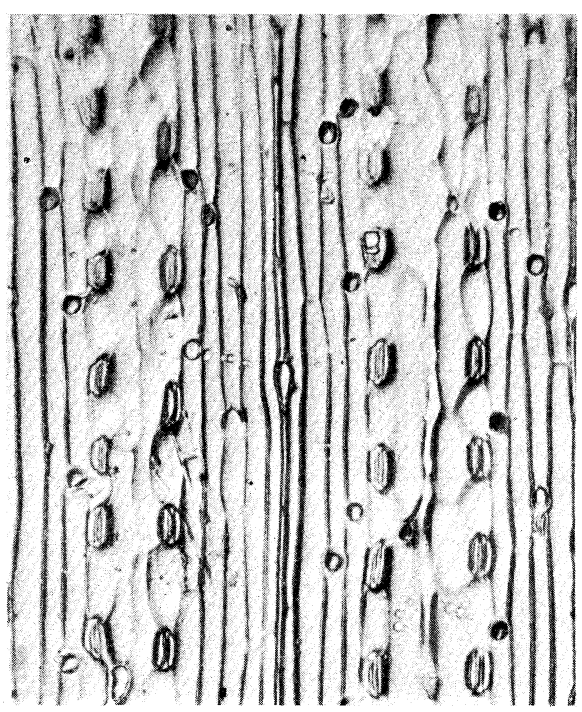

26

Abb. 25. Epidermis von Haplovulgare (Mikrophotographie eine Danerpräparats nach S. U. M. P.) Vergr. ca. 90-fach. Abb. 26. Wie Abb. 25. Diplovulgare.

\section{Diskussion.}

Im J. 1930 haben Kinara und Nishiyama festgestellt, dass von den 3 vulgare-Genomen $\mathrm{A}, \mathrm{B}$ und $\mathrm{D}, \mathrm{A}$ und $\mathrm{B}$ in 3 und $\mathrm{B}$ und $\mathrm{D}$ in $2-3$ Gliedern sich paaren können. Diesen an Bastarden gewonnenen Befund konnte KiHara (1936) für die Genome A und B auf Grund der Reifungsteilungen von Haplodurum bestätigen. Bei Haplovulgare waren 5-6 Bindungen (in Gestalt von Bi- bzw. Tripartiten) zu erwarten. Yamasaki (1935) gibt 4 als höchste gefundene Bipartitenzahl an, ich konnte nicht mehr als 3 finden. Nach meinen Beobachtungen weisen die Bipartiten aber verschiedene Gestalt auf. Wenn die in Abb. 10 gebrachten Unterschiede zwischen den 6 heteromorphen Bipartiten zuverlässig sind und zu dem Schluss berechtigen, dass die zu den betreffenden Paaren zusammentretenden Chromosomen alle oder fast alle verschiedenen sind, so könnte man daraus folgern, dass bei meinem Haplovulgare unter günstigen Bedingungen für 
die Paarung ca. 6 Bindungen in einer P.M.Z. vorkommen könnten. Die Tripartiten sind am einfachsten auf die Paarung zwischen den 3 VulgareGenomen zurückzuführen.

Betreffs der Entstehung der haploiden Zwillingspflanze möchte ich mich vorläufig Kinara (1936) anschliessen und annehmen, dass eine befruchtete Synergide sich zur diploiden und die unbefruchtete Eizelle zur haploiden Zygote entwickelt hat. Im Lichte dieser Auffassung sind die haplo-diplo-Zwillinge eingonig. KAPPERT (1933) hat für seinen Fall bei Linum einwandfrei eingonige Herkunft solcher Zwillingspaare nachgewiesen. Ramian (et alii, 1935) hat auf genetischem Wege festgestellt, dass seine Albino-Grüne-Paare bei Oryøa zweigonig waren. Es wäre von grösserem Interesse zu erfahren, ob auch diese Zwillingspaare haplo-diplo oder diplo-diplo-Paare gewesen sind, da die Vorstellung der Zwillingeu Entstehung von haplo-diplo-Zwillingen auf Schwierigkeiten stösst.

\section{Zusammenfassung.}

Es wurde bei Triticum vulgare ein haplo-diploides Zwillingspaar gefunden. Der Verlauf der Reifungsteilungen bei dem haploiden Individuum gestaltet sich wie bei Triticum-Bastarden mit vielen Univalenten. Die Befunde betreffs der Bindungen in der I. Metaphase stehen in Einklag. mit den Ergebnissen der genomanalytischen Untersuchungen von KiHARA.

Diese Untersuchungen wurden auf Anregung und unter Leitung von Herrn Prof. Dr. H. Kinara ausgeführt, dem ich auch an dieser Stelle für alle Hilf herzlich danke.

\section{Laboratorium für Vererbungslehre, Biologisches Institut, Kyoto Kaiserliche Universität.}

\section{Literaturverzeichnis.}

CHIZAKI, Y. 1934. Another new haploid plant in Triticum monococcum L. Bot. Mag. Tokyo 48: 621-628.

Gaines, E. F., and H. C. Aase. 1926. A haploid wheat plant. Amer. Journ. Bot. 13: 81-90.

KAPPERT, H. 1933. Erbliche Polyembryonie bei Linum usitatissimum. Biol. Zbl. 53: $276-307$.

Katayama, Y. 1934a. Haploid formation by $X$-rays in Triticum monococcum. Cytologia 5: 235-237.

- 1934b. Experimental formation of haploid plants in Triticum monococcum. (japanisch mit engl. Résumé). Bull. Almuni Assoc. Utsunomiya Agr. Coll. 1: $41-50$.

- 1935. Karyological Comparisons of haploid plants from octoploid Aegilotricum and diploid wheat. Jap. Journ. Bot. 7: 349-380.

Kinara, H. 1936. Ein diplo-haploides Zwillingspaar bei Triticum aurum. (japanisch 
mit deutsch. Résumé). Agric. \& Hort. Tokyo 11: 1425-1433.

Kihara, H. und Y. Katayama. 1932. Über das Vorkommen von haploiden Pflanzen bei Triticum monococcum. (japanisch mit deutsch. Résumé). Kwagaku, Tokyo 2: 408-410.

— 1933. Reifungsteilungen bei dem haploiden Triticum monococcum. (japanisch mit deutsch. Résumé). Agric. \& Hort. Tokyo 8: 1-17.

Kinara, H. und I. Nishiyama. 1930. Genomanalyse bei Triticum und Aegilops. I. Cytologia 1: 263-284.

Nuкилima, G. 1935. Occurence of a haploid in Triticum turgidum. Jap. Journ. Gen. 11: $246-247$.

Namikawa, S. and J. KAwaKami. 1934. On the oceurence of haploid, triploid and tetraploid plants in twin seedlings of common wheat. Proc. Imp. Acad. Tokyo 10 : $668-671$.

Ramiah, K., N. Parthasarthi and S. Rananujan. 1933. Haploid plant in rice (Oryza sativa). Current Sci. Mysore 1: 277-278.

—_ 1935. Polyembryony in rice (Oryza sativa). Indian Journ. Agr. Soc. 5: 119124.

YAMASAKI, Y. 1934. The haploid plant of common wheat, Triticum vulgare. Host. Cytologia 5: 306-307.

1935. Cytological studies on haploid wheat plants. (japanisch). Jap. Journ. Gen. 11: $314-315$. 